\title{
ENERGY AND PROPULSION OPTIMIZATION OF SOLID- PROPELLANT GRAIN OF A HYBRID POWER DEVICE
}

\author{
Sergey S. Bondarchuk ${ }^{1,2}$, lliya S. Bondarchuk ${ }^{1}$, Boris V. Borisov ${ }^{3, \text { a }}$, Alexandr S. Zhukov ${ }^{1}$ \\ ${ }^{1}$ Tomsk State University, 634050 Tomsk, Russia \\ ${ }^{2}$ Tomsk State Pedagogical University, 634061 Tomsk, Russia \\ ${ }^{3}$ National Research Tomsk Polytechnic University, 634050 Tomsk, Russia
}

\begin{abstract}
A method of distribution of an additional solid-phase component (oxidizer) providing uniformity of grain burning for the purpose of evaluation and optimization of energy and propulsion parameters of hybrid solid-propellant motor is proposed in the paper.
\end{abstract}

\section{Introduction}

Non-uniform character of fuel grain burnout along its length is a typical disadvantage of hybrid solidpropellant power devices [1]. Due to oxidizer consumption for combustion reaction the density of oxidizer flow varies along the surface of grain channel. The most intensive burning takes place in the area of a front face of the propellant grain as for the area down the flow a high-temperature channel erosion takes place in parallel to combustion reaction. Therefore, a carry-over of unreacted fuel is possible due to insufficient amount of oxidizer. All of the above leads to reduction of thrust due to incompleteness of combustion of fuel mixture.

To evaluate and optimize energy and propulsion parameters of the motor we considered "fueloxidizer" balance equations for fuel burning rate in the form of power-law dependence on oxidizer flow density. In this case the change of oxidizer flow along the grain channel of a hybrid motor can be described by the following equation.

$$
\frac{d}{d x}(\varrho u S)=-\Pi \alpha_{o x} \varrho_{f} a(\varrho u)^{v}
$$

where $\mathrm{Q}, \mathrm{u}$ - oxidizer density and flow rate;

$S, \Pi-$ flow area and perimeter of cylindrical channel with radius $r$;

$a, v$ - burning rate law constants; $\mathrm{Q}_{f}$ - fuel density;

$\alpha_{\mathrm{ox}}=m_{o x} / m_{f}$ - ratio between "consumed" oxidizer mass $m_{o x}$ and fuel mass $m_{f}$ in the process of burning. In case of round cylindrical channel with $S=$ const the solution of equation (1) for oxidizer flow at an arbitrary point (section) of the channel is as follows

$$
\left.(\varrho u)\right|_{x}=\left[(\varrho u)_{0}^{1-v}-\varphi x\right]^{1 /(1-v)}, \varphi=\frac{2}{r} \alpha_{o x} \varrho_{f}(1-v) a
$$

where $(\varrho u)_{0}=m_{o x}^{i n} / S-$ oxidizer "injection" mass flux ( $m_{o x}^{\text {in }}-$ total oxidizer flow rate).

\footnotetext{
a Corresponding author: bvborisov@tpu.ru
} 
Based upon equation (2) the amount of oxidizer m_ox used for burning can be determined from the following relationship

$$
m_{o x}=\left\{\begin{array}{l}
m_{o x}^{i n}-S \cdot\left[(\varrho u)_{0}^{1-v}-\varphi L\right]^{1 /(1-v)}, \operatorname{ec\lambda u}(\varrho u)_{0}^{1-v}>\varphi L, \\
m_{o x}^{i n}, e c \mathcal{u} u(\varrho u)_{0}^{1-v} \leq \varphi L .
\end{array}\right.
$$

The mass of fuel $m_{f}$ consumed by burning process comprises $m_{f}=m_{o x} / \alpha_{o x}$ and, therefore, total mass of the mixture of combustion products and residual (unused) oxidizer will be equal to $m_{\Sigma}=m_{o x}^{i n}+m_{f}$.

Minimal oxidizer flow density $m_{o x}^{\min }$ necessary for grain burning along the whole its length can be evaluated using equation (2), i.e. when $\left.(\varrho u)\right|_{x=L}=0$ :

$$
m_{o x}^{\min }=(\varphi L)^{1-v}
$$

Thermodynamic characteristics of the mixture (gas constant $R$ and isobaric heat capacity $C_{p}$ ) for a known value of $m_{o x}^{i n}$ are determined additively [2] using individual characteristics of gas, oxidizer $\left(R_{o x}, C_{p o x}\right)$ and combustion products $\left(R_{f}, C_{p_{f}}\right): R=c_{o x} R_{o x}+c_{f} R_{f}, C_{p}=c_{o x} C_{p_{o x}}+c_{f} C_{p_{f}}$, where mass fractions of residual (unused) oxidizer $c_{o x}$ and combustion products $c_{f}$ are determined by the following relationships:

$$
c_{o x}=\frac{m_{o x}^{i n}-m_{o x}}{m_{\Sigma}}, c_{f}=\frac{m_{f}+m_{o x}}{m_{\Sigma}}=1-c_{o x} .
$$

The temperature $T$ of the mixture of combustion products and "unused" oxidizer can be determined using the law of conservation of energy

$$
\frac{\gamma}{\gamma-1} R T m_{\Sigma}=\left(m_{f}+m_{o x}\right)\left(\frac{\gamma}{\gamma-1} R T\right)_{f}-m_{o x}^{i n}\left(\frac{\gamma}{\gamma-1} R\right)_{o x}\left(\tilde{T}-T_{o x}\right)
$$

The first member of the right part of equation (5) describes energy input due to fuel combustion products joining the flow with temperature $T_{f}$. The last member of the right part of this equation represents energy losses associated with oxidizer gas heating from its initial temperature $T_{o x}$ to a certain average value $\tilde{T}$. At a first approximation can be taken as $\tilde{T}=\left(T_{f}+T_{o x}\right) / 2$.

Then, using the mass balance between the gas fed to the combustion chamber $m_{o x}^{\text {in }}$ in and combustion products formed $m_{\Sigma}$ considering the fact that they are equal to flow rate $G$ trough the nozzle cluster with throat section $S_{*}$ for the case of critical flow

$$
G=S_{*} \Gamma(\gamma) \cdot \frac{p}{\sqrt{R T}}=m_{\Sigma}, \quad \Gamma(\gamma)=\sqrt{\gamma\left(\frac{2}{\gamma+1}\right)^{\frac{\gamma+1}{\gamma-1}}},
$$

pressure $p$ in combustion chamber and $P$ engine thrust can be determined

$$
p=\frac{m_{\Sigma}}{S_{*} \Gamma(\gamma)} \sqrt{R T}, P=(\gamma+1)\left(\frac{2}{\gamma+1}\right)^{\frac{\gamma}{\gamma-1}} S_{*} p .
$$

In case if thrust value is less than required, the desired level can be achieved by means of increase of oxidizer flow rate $m_{o x}^{i n}$ this, however, will increase the amount of residual (unused) oxidizer. The method of adding an oxidizer component to the solid-propellant grain with the oxidizer mass fraction $z(x)$ increasing along the grain length towards the nozzle cluster according to equation [3] can be used for optimization of energy and propulsion parameters of the motor neutralizing the abovementioned disadvantage of the process. 


$$
z(x)=\frac{f(x)}{1+f(x)}, \quad f(x)=\beta\left[1-\left(1-\varphi(\varrho u)_{0}^{v-1} x\right)^{\frac{1}{1-v}}\right],
$$

where $\beta$ - ratio of the mass of additional oxidizer "consumed for burning" to fuel mass.

Equation (4) was obtained by means of simple algebraic transformations of (2) and (3) to provide the uniformity of fuel burnout profile.

A hybrid power device with the following parameters: grain length $L=1 \mathrm{~m}$; channel radius $r=0.1 \mathrm{~m}$; oxidizer mass flow rate $m_{o x}^{i n}=10 \mathrm{~kg} / \mathrm{s}$; fuel density $\varrho_{f}=1600 \mathrm{~kg} / \mathrm{m}$; burning rate law: $a=0.0127 \mathrm{~mm} / \mathrm{s}, v=0.65$ was used as an example.

Let us consider the composition containing $85 \%$ of inert fuel-binder (FB) - butadiene rubber, plasticized by transformer oil and $15 \%$ of Al powder ASD-4 (oxidizer - gaseous oxygen) as a solidfuel material (SFM) for a solid-propellant grain. Data for fuels analysed with various types of additional oxidizer are given in Table 1.

Table 1. Fuel blend composition.

\begin{tabular}{|c|c|c|c|c|c|}
\hline \multirow{2}{*}{} & \multicolumn{4}{|c|}{ Content, wt\% } & \\
\cline { 2 - 6 } & Additional oxidizer & FB & Aluminum & SFM & $\beta$ \\
\hline 1 & Ammonium perchlorate, 89.6 & 8.8 & 1.6 & 10.4 & 8.61 \\
\hline 2 & Potassium perchlorate, 89.6 & 11.6 & 2.0 & 13.6 & 6.35 \\
\hline 3 & Nitronium perchlorate 56.2 & 37.2 & 6.6 & 43.8 & 1.28 \\
\hline
\end{tabular}

To provide uniform burning of solid-propellant grain along its length a solid oxidizer (AP, PP, NP) was added to the composition. Oxidizer component mass fraction distribution along the grain length calculated using equation (4) is shown in Fig.1 for ammonium perchlorate (AP curve), potassium perchlorate (PP curve) and nitronium perchlorate (NP curve).

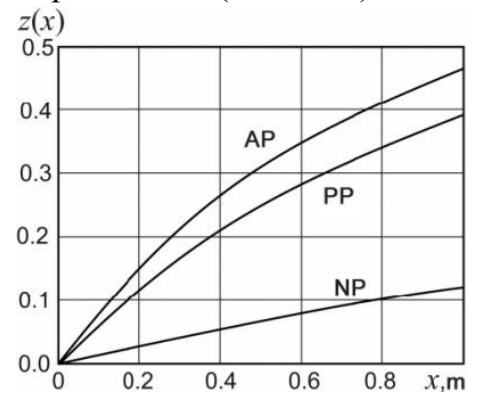

Figure 1. Oxidizer component mass fraction distribution.

As it can be seen from the graph the content of oxidizer component in solid-propellant grain composition must increase monotonically from zero (at the head section of the grain) to a maximum value $(z=0.465$ - for ammonium perchlorate, $z=0.390$ - for potassium perchlorate, $z=0.114-$ for nitronium perchlorate) in order to provide optimal uniform burnout. In this case grain burns uniformly along the channel axis which provides high combustion completeness and, therefore, high thrustweight ratio.

Financial support of this research by the Ministry of Education and Science of the Russian Federation under the Federal Target Program "Research and development on priority directions of scientific-technological complex of Russia for 2014-2020" (agreement № 14.578.21.0034, unique 
identifier PNI RFMEFI57814X0034) and "The Tomsk State University Academic D.I. Mendeleev Fund Program” grant № 8.2.46.2015 is gratefully acknowledged.

\section{References}

1. S.S. Bondarchuk, A.B. Vorozhtsov, A.S. Zhukov, B.V. Borisov, Russ. Phys. J., 57, (12), 1796 (2015)

2. S.S. Bondarchuk, A.B. Vorozhtsov, E.A. Kozlov, Y.V. Feshchenko,J. Propul. Power, 11 (4), 593 (1995).

3. V.A. Arkhipov, S.S. Bondarchuk, A.S. Zhukov, B.V. Pevchenko, L.A. Savelyeva. Hybrid rocket motor. Patent application No 2014149746. Priority dated 09.12.2014. (In Russian) 